psychopraxis. neuropraxis $2022 \cdot 25: 26-31$ https://doi.org/10.1007/s00739-021-00773-6 Angenommen: 3. Dezember 2021 Online publiziert: 1. Februar 2022

(c) Der/die Autor(en) 2022

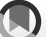

\section{Reinhold Schmidt}

Universitätsklinik für Neurologie, Medizinische Universität Graz, Graz, Österreich

\section{Palliative Aspekte bei Demenz} zur Palliation bei Demenz gibt es kaum. Die wichtigste Richtlinie ist das von der European Association of Palliative Care erstellte White Paper [1].

Es wurde als Delphi-Studie entwickelt und ist hauptsächlich Gegenstand dieses Überblicks. Expertinnen und Experten zum Thema Demenz wurden eingeladen, ihre Meinung zu bestimmten Themen bei diesen Krankheitsbildern abzugeben. Diese wurden dann gesammelt und gereiht sowie in mehreren Stufen zu einer Empfehlung ausgearbeitet. Im Gegensatz zur sonstigen Guideline-Erstellung beruht diese also nicht auf Evidenz, sondern auf Expert ${ }^{*}$ innenmeinungen.

Der Relevanz und Wichtigkeit dieses Dokuments tut dies jedoch keinen Ab-

\section{Infobox 1 Empfehlungen zur Domäne 2}

Personenzentrierte Pflege und Kommunikation, gemeinsame Entscheidungsfindung

- Betrachtung der Probleme in der Pflege aus Patient*innenperspektive

- Gemeinsame Entscheidungsfindung mit Patient*in und Betreuer*in ("caregiver")

- Betreuungsteam tritt an die Patientin bzw. den Patienten und deren/dessen Familie heran hinsichtlich Verlauf, palliativer Versorgung und Einbeziehung in die Versorgung

- Reaktion auf spezifische Bedürfnisse der Patientin/des Patienten und ihrer/ seiner Familie sind im Krankheitsverlauf entscheidend

- Zum Ausdruck gebrachte Präferenzen hinsichtlich der Betreuungssituation sind prinzipiell zu berücksichtigen, aber abzuwägen mit Fragen der Sicherheit und Belastung der Caregiver

- Innerhalb des multidisziplinären Teams sollte regelmäßig über auftretende Schwierigkeiten für die Patientin/den Patienten und ihre/seine Familie diskutiert werden
Flüssigkeitssubstitution. Aber Guidelines bruch. An dem Prozess arbeiteten 64 Experten aus 23 Ländern (•Tab. 1). Es wurde 11 Domänen spezifiziert, die für dieses Thema wichtig sind. Diese mündeten dann in 57 Empfehlungen. @ Abb. 1 gibt diese 11 Domänen wieder. Die Ergebnisse spiegeln auch die kulturellen Unterschiede der 23 Länder beim Zugang zu diesem Thema wider.

\section{》) Die Einschätzung zur Wichtigkeit erfolgte im Rahmen einer Delphi-Studie}

Die rot umrandeten Bereiche erschienen den Teilnehmern als besonders wichtig. Der höchste Wert war der für „optimal treatment of symptoms and providing

\section{Infobox 2 Empfehlungen zur Domäne 6}

Vermeidung von aggressiver, belastender und sinnloser Behandlung

- Spitalstransport sorgfältig unter Berücksichtigung der Pflegeziele abwägen

- Begleitmedikation regelmäßig im Hinblick auf Pflegeziele und Nebenwirkungen überprüfen

- Möglichst keine freiheitsbeschränkenden Maßnahmen

- Flüssigkeitszufuhr subkutan, z. B. im Fall einer Infektion, nicht in der Sterbephase (nur moderate Übereinstimmung)

- Permanente Sondenernährung vermeiden. Nahrungszufuhr durch Hand zu bevorzugen (nur moderate Übereinstimmung)

- Antibiotika im Rahmen von Infektionen zur Erhöhung des Wohlbefindens. Lebensverlängernde Effekte sind vor allem bei Behandlungsentscheidungen in Zusammenhang mit Pneumonien abzuwägen 
comfort“. Weniger bedeutend, aber immer noch als wichtig eingestuft, wurde die Frage, ob für Demenz das Palliativkonzept überhaupt Gültigkeit haben soll. Wann soll es zum Einsatz kommen? Ist es wichtig, schon bei der Diagnosestellung einer Demenz damit zu arbeiten oder erst in späteren Stadien? Interessanterweise haben hier die Teilnehmer keine absolute Übereinstimmung. Im Gegensatz zu anderen Erkrankungen zeigt die Demenz im letzten Lebensjahr wenig Beeinträchtigung. Dies unterscheidet diese Erkrankung wesentlich. Die Verläufe im letzten Lebensjahr bei unterschiedlichen Erkrankungen sind sehr heterogen. In einer Untersuchung im New England Journal wurden diese klassifiziert in „no disability", "persistant severe disability“ und „catastrophic disability“ [2]. Im Gegensatz zu einer hohen Rate an „catastrophic disabilty“ bei Krebskranken zeigen Demenzkranke zumeist „no disability“. Möglicherweise erklärt dies die unterschiedlichen Zugänge der Experten zur Frage, wann Palliative Care bei Demenz beginnen soll. Prinzipiell wird eine Palliativgesellschaft jedoch die Tendenz haben, möglichst früh begleitend in einem Krankheitsprozess zu beginnen.

Aufgrund der langsamen Verschlechterung bei fortgeschrittener Demenz und weil sie eine terminale Erkrankung ist, sind die Experten der Meinung, dass das Palliativkonzept hier anzuwenden ist, wobei die Behandlungsprioritäten in den unterschiedlichen Phasen der Demenz jedoch verschiedene Schwerpunkte haben. Das Palliativkonzept beginnt nach allgemeiner Einschätzung erst in den mittleren Stadien zu tragen. Es gibt also einen prinzipiellen Konsens darüber, dass aufgrund der Progredienz Demenz einer palliativen Pflege zugänglich sein sollte. Ab wann dies geschehen soll, wird unterschiedlich bewertet. Einigkeit bestand darüber, dass die palliative Pflege bei fortschreitender Erkrankung immer mehr an Bedeutung gewinnt und in schweren Stadien auch das Hospizkonzept zum Tragen kommen sollte. Als sehr wichtig eingeschätzt wurden die Punkte personifizierte Pflege und gemeinsame Entscheidungsfindung (•Infobox 1). Die Herausforderung besteht darin, all diese Empfehlungen, die hier so selbstver- psychopraxis. neuropraxis $2022 \cdot 25: 26-31 \quad$ https://doi.org/10.1007/s00739-021-00773-6 (c) Der/die Autor(en) 2022

\section{R. Schmidt \\ Palliative Aspekte bei Demenz}

\section{Zusammenfassung}

Die Frage, wie viel Palliativmedizin Demenzkranke brauchen und wann diese zum Einsatz kommen soll, sind die ersten beiden Fragen, die sich aufdrängen, wenn man sich diesem Thema bei dieser Erkrankung nähert. Die Frage "ob" ist leicht und schnell mit ja beantwortet. Die Frage "wann" ist deutlich komplexer. Studien zu diesem Thema sind spärlich und basieren eher auf Expertenmeinungen als auf Zahlen und Daten (Stichwort Delphi-Studien). Die daraus resultierenden Informationen sind

\section{Palliative Aspects of Dementia}

\section{Abstract}

While there is no doubt that people with dementia might profit from palliative strategies, the time point when to start however is still debated. Clinical studies providing information about this question are lacking. Information is based more on expert opinion formulations sampled and published in the form of Delphi studies. However, the information gathered is nonetheless important. The answers to predefined questions are accurate and informative. The jedoch nicht weniger wichtig, nicht weniger akkurat. Die Tatsache, dass Expertinnen und Experten von unterschiedlichen Ländern zur Meinungsgebung eingeladen sind, spiegelt in hohem Maße auch die kulturellen Unterschiede im Zugang speziell bei dieser Erkrankung wider. Der vorliegende Beitrag gibt einen Überblick zu diesem Thema.

\section{Schlüsselwörter}

Demenz · Palliative Care · Delphi-Studie . Vorsorge $\cdot$ Wohlbefinden fact that experts from all over the world have given their opinions also shows very different points of view, which vary due to different cultural influences and aspects regarding dementia. This article provides an overview on this important topic.

\section{Keywords}

Dementia - Palliative care · Delphi studies . Prevention - Comfort of care ständlich formuliert scheinen, im Alltag auch umzusetzen.

Des Weiteren wird Domäne 6 als sehr wichtig hervorgehoben, in der es um die Vermeidung aggressiver, belastender und sinnloser Behandlungsstrategien geht. Auch hier wurden Empfehlungen formuliert. Infobox 2 gibt diese wieder. Generell steht bei der Entscheidung über eine Behandlung als Ziel die Lebensqualität und nicht die Lebensverlängerung im Vordergrund.

\section{》) Besonderer Punkt: \\ Vermeidung aggressiver und be- lastender Behandlungsstrategien}

Ein großes Ziel bei Demenzpatienten muss die Schaffung maximal möglicher Lebensqualität sein und die Findung eines Zustands mit möglichst wenig „dyscomfort“ im täglichen Leben. Bei den Experten wurde dies in Domäne 7 abgefragt. Die Empfehlungen dazu gibt

\section{- Infobox 3}

Nicht explizit enthalten und doch Teil dieses Themas ist die Frage nach einer Sondenimplantation und Sondenernährung. Die zu dieser Behandlung vorliegenden Studien weisen ein hohes $\mathrm{Ne}$ benwirkungsprofil nach. Auch Messungen mit Dyscomfort-Skalen wie der Alzheimer Dyscomfort Scale belegen, dass sich das Wohlbefinden bessert, sobald von Sondenernährung oder Flüssigkeitsersatz Abstand genommen wird. Erweitert gehören hier hin auch die Evaluierung von Schmerz und neu aufgetretenen Verhaltensauffälligkeiten als indirekte Zeichen generellen Unwohlseins. Zur Evaluierung stehen für Demenz erarbeitete Skalen zur Verfügung, sodass zumindest zu einem gewissen Maß objektive Beurteilungsparameter gewonnen werden können. Ein zusätzliches Anfordern 


\begin{tabular}{|c|c|c|c|c|c|}
\hline \multirow[t]{2}{*}{ Domain } & \multirow{2}{*}{$\begin{array}{l}\text { Importance rating,a } \\
\text { mean }(S D) \\
\text { Round } 2(n=64) \text { and, } \\
\text { if applicable, round } 3 \\
(n=59)\end{array}$} & \multirow[t]{2}{*}{$\begin{array}{l}\text { Rank number } \\
\text { importance }\end{array}$} & \multirow{2}{*}{$\begin{array}{l}\text { Priority for research } \\
\text { rank, }{ }^{b} \text { mean }(S D) \\
\text { Round } 3, n=55^{c}\end{array}$} & \multirow[t]{2}{*}{$\begin{array}{l}\text { Rank number } \\
\text { priority for } \\
\text { research }\end{array}$} & \multirow[t]{2}{*}{$\begin{array}{l}\text { Correlation rank } \\
\text { numbers: Spearman } \\
\text { coefficient }^{\mathrm{d}} \text { ( } \mathrm{p} \text {-value) }\end{array}$} \\
\hline & & & & & \\
\hline $\begin{array}{l}\text { Overall rating for the importance of the } \\
\text { set of recommendations }\end{array}$ & $8.9(1.2)$ & N/A & N/A & N/A & - \\
\hline I. Applicability of palliative care & $\begin{array}{l}8.3(1.9), \text { round } 2 \\
8.4(1.9) \text {, round } 3\end{array}$ & 10 & $5.3(3.8)$ & 4 & $+0.03(0.83)$ \\
\hline $\begin{array}{l}\text { 2. Person-centred care, communication } \\
\text { and shared decision-making }\end{array}$ & $9.3(1.1)$ & 2 & $7.6(2.6)$ & 1 & $-0.15(0.26)$ \\
\hline $\begin{array}{l}\text { 3. Setting care goals and advance } \\
\text { planning }\end{array}$ & $8.8(1.4)$ & 9 & $6.3(2.9)$ & 3 & $-0.006(0.97)$ \\
\hline 4. Continuity of care & $8.9(1.4)$ & 8 & $5.0(2.8)$ & 7 & $-0.11(0.42)$ \\
\hline $\begin{array}{l}\text { 5. Prognostication and timely } \\
\text { recognition of dying }\end{array}$ & $\begin{array}{l}7.3(2.2), \text { round } 2 \\
8.0(1.5) \text {, round } 3\end{array}$ & 11 & $3.9(3.2)$ & 8 & $+0.44(0.001)$ \\
\hline $\begin{array}{l}\text { 6. Avoiding overly aggressive, } \\
\text { burdensome or futile treatment }\end{array}$ & $9.1(1.6)$ & 5 & $5.2(3.0)$ & 5 & $+0.29(0.03)$ \\
\hline $\begin{array}{l}\text { 7. Optimal treatment of symptoms and } \\
\text { providing comfort }\end{array}$ & $9.4(1.1)$ & 1 & $6.7(2.4)$ & 2 & $+0.21(0.13)$ \\
\hline 8. Psychosocial and spiritual support & $8.9(1.3)$ & 7 & $3.8(2.2)$ & 10 & $+0.17(0.21)$ \\
\hline 9. Family care and involvement & $9.2(1.1)$ & 3 & $5.1(2.3)$ & 6 & $+0.15(0.27)$ \\
\hline 10. Education of the health-care team & $9.0(1.3)$ & 6 & $3.9(2.6)$ & 9 & $-0.005(0.97)$ \\
\hline II. Societal and ethical issues & $9.2(1.2)$ & 4 & $2.2(3.1)$ & 11 & $-0.10(0.48)$ \\
\hline
\end{tabular}

Abb. 1 \ Von Expertinnen und Experten als wichtig erachtete Themen für die Palliativversorgung bei Demenz und für die Einstufung von Prioritäten in der Forschung [1, S. 197-209]

\section{Infobox 3 Empfehlungen zur Domäne 7}

Beste Behandlung von Symptomen und Schaffen von Wohlbefinden

- Ein ganzheitlicher Zugang hinsichtlich der Behandlung von Symptomen ist entscheidend, da Symptome häufig miteinander in Beziehung stehen oder unterschiedlich ausgedrückt werden (z.B. Schmerz als Agitiertheit)

- Um Symptomursachen zu unterscheiden, kann der Caregiver wichtig sein (z. B. Schmerz oder Frieren)

- Instrument zur Erfassung von Schmerz, Unwohlsein und Verhalten sollten für Screening und Monitoring zum Einsatz kommen

- Nichtpharmakologisch und pharmakologisch anlassbezogen therapieren

- Spezialisierte Pflegeteams als Unterstützung bei spezifischen Symptomen (u.U Demenz-Expertise zusätzlich erforderlich)

und Hinzuziehen von Demenzexpertise ist hier sinnvoll, im Alltag jedoch nicht leicht $\mathrm{zu}$ organisieren.

Letztendlich wurden auch die äußerst wichtigen Bereiche Betreuung durch die Familie, Einbeziehung der Familie und
Einbeziehung des Umfelds ebenso abgefragt, wie Ausbildung des Behandlungsteams, gesellschaftliche Fragen, ethische Fragen und die Belastung der Caregiver. Besonders gefordert werden diese Gruppen zuerst bei der Stellung der Diagnose. Danach dann, wenn Patienten z. B. ein herausforderndes Verhalten entwickeln oder ein Krankenhausaufenthalt notwendig wird. Und letzten Endes natürlich beim Ableben der geliebten Person.

Hier sind Informationen zur Erkrankung ganz entscheidend. In der Rolle als Entscheidungsträger brauchen die $\mathrm{Be}$ treuer Unterstützung. Auch juridischer Beistand ist sinnvoll. Die Betreuenden müssen Ausbildung in Palliativ Care mit spezieller Berücksichtigung der Bedürfnisse Demenzkranker erhalten. Der Demenzplan für Österreich greift hier sicher noch zu kurz.

Hervorzuheben wäre letztendlich noch die Möglichkeit, ja die Notwendigkeit der Patientenverfügung und/oder Vorsorgevollmacht. Es ist gerade im Falle von Demenzerkrankungen besonders wichtig, solche Willensbekundungen $\mathrm{zu}$ formulieren, solange es der geistige $\mathrm{Zu}$ stand noch zulässt. Dies wäre ein Grund, professionelle palliative Betreuung früh hinzuzuziehen, denn meist kommt der Tipp, seine Dinge zu regeln, von den $\mathrm{Be}$ treuern aus dieser Profession. $\bullet$ Infobox 4 listet eine Auswahl der Empfehlungen zu dieser Domänen auf.

\section{Fazit für die Praxis}

- Nach übereinstimmender Expertenmeinung sollte Palliative Care in die Betreuung Demenzkranker integriert sein.

- Für diese Betreuung lässt sich aus mehreren Gründen argumentieren. Zum einen sind die Symptome bei Demenz vielfältig und komplex. Eine Betreuung durch ein multidisziplinäres Team ist sehr von Vorteil. Zum anderen ist die Demenz letztendlich eine terminale Erkrankung, auch wenn sie über viele Jahre verlaufen kann.

- Im Gegensatz zum generellen Konsens, dass Palliative Care bei Demenz 
Hier steht eine Anzeige.

\section{曾 Springer}


Hier steht eine Anzeige. 照 Springer

\section{Infobox 4 Ausgewählte Empfeh-} lungen zu den Domänen 9-11

Betreuung durch und Einbeziehung der Familie, Ausbildung des Behandlungsteams, Gesellschaft und Ethik

- Familien-Caregiver-Belastung: Unterstützung im Verlauf (Diagnosestellung, herausforderndes Verhalten, Institutionalisierung, massive Verschlechterung, nahender Tod), Information zur Erkrankung, Unterstützung in der Rolle als Entscheidungsträger, als Hinterbliebene nach einer meist langen Zeit der Pflege

- Ausbildung des Pflegeteams: Alle Beteiligten müssen Fähigkeiten in der palliativen Betreuung bei Demenz besitzen

- Gesellschaft und Ethik: Verfügbarkeit palliativer Betreuung für alle Demenzkranken ebenso wie für andere Krankheiten, Ausbildungscurricula, ökonomische und systemische Anreize, um zur Endof-Life Care zu ermutigen, nationale Demenzstrategien sollen Palliative Care, End-of-Life Care und Langzeitbetreuung beinhalten. Vice versa sollten Palliativund Langzeitversorgungseinrichtungen Demenz mit einbeziehen

- Österreich: Handlungsempfehlung 5a "Bestmögliche Langzeitbetreuung von Menschen mit Demenz"

- Qualifizierte Versorgung demenziell Erkrankter in den Bereichen palliative Versorgung und Hospiz

sinnvoll ist, ist der Zeitpunkt des Beginns sehr unterschiedlich diskutiert.

\section{Korrespondenzadresse}

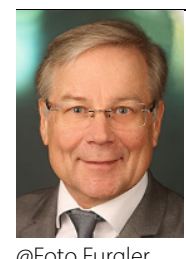

Univ. Prof. Dr.

Reinhold Schmidt

Universitätsklinik für

Neurologie, Medizinische

Universität Graz

Auenbruggerplatz 22,

8036 Graz, Österreich

reinhold.schmidt@

medunigraz.at

Funding. Open access funding provided by Medical University of Graz.

\section{Einhaltung ethischer Richtlinien}

Interessenkonflikt. R. Schmidt gibt an, dass kein Interessenkonflikt besteht.

Für diesen Beitrag wurden von den Autoren keine Studien an Menschen oder Tieren durchgeführt.
Für die aufgeführten Studien gelten die jeweils dort angegebenen ethischen Richtlinien.

Open Access. Dieser Artikel wird unter der Creative Commons Namensnennung 4.0 International Lizenz veröffentlicht, welche die Nutzung, Vervielfältigung Bearbeitung, Verbreitung und Wiedergabe in jeglichem Medium und Format erlaubt, sofern Sie den/die ursprünglichen Autor(en) und die Quelle ordnungsgemäß nennen, einen Link zur Creative Commons Lizenz beifügen und angeben, ob Änderungen vorgenommen wurden.

Die in diesem Artikel enthaltenen Bilder und sonstiges Drittmaterial unterliegen ebenfalls der genannten Creative Commons Lizenz, sofern sich aus der Abbildungslegende nichts anderes ergibt. Sofern das betreffende Material nicht unter der genannten Creative Commons Lizenz steht und die betreffende Handlung nicht nach gesetzlichen Vorschriften erlaubt ist, ist für die oben aufgeführten Weiterverwendungen des Materials die Einwilligung des jeweiligen Rechteinhabers einzuholen.

Weitere Details zur Lizenz entnehmen Sie bitte der Lizenzinformation auf http://creativecommons.org/ licenses/by/4.0/deed.de.

\section{Literatur}

1. van der Steen JT, Radbruch L, Hertogh CM et al (2014) White paper defining optimal palliative care in older people with dementia: a Delphi study and recommendations from the European association of palliative care. Palliat Care 28(3):197

2. Gill TM et al (2010) Trajectories of disability in the last year of life. N Engl J Med 362:1173

Hinweis des Verlags. Der Verlag bleibt in Hinblick auf geografische Zuordnungen und Gebietsbezeichnungen in veröffentlichten Karten und Institutsadressen neutral. 
Hier steht eine Anzeige.

\section{曾 Springer}

